\title{
ON PROXIMINAL SETS OF NORMAL OPERATORS
}

\author{
DONALD D. ROGERS ${ }^{1}$
}

\begin{abstract}
It is shown that neither the set of normal operators nor the set of orthogonal projections is a proximinal subset of the space of bounded operators on an infinite-dimensional Hilbert space.
\end{abstract}

Let $H$ denote a separable complex Hilbert space with inner product ( , ) and norm $\|\cdot\|$; let $B(H)$ denote the space of bounded linear operators on $H$, with the usual operator norm, also denoted by $\|\cdot\|$. A subset $\delta$ of $B(H)$ is called proximinal in $B(H)$ if for every $T$ in $B(H)$ there exists at least one $X_{\mathrm{o}}$ in $\delta$ such that $\left\|T-X_{\mathrm{o}}\right\|=\inf \{\|T-X\|: X$ in $\delta\}$; such an operator $X_{\mathrm{o}}$ is called an $\delta$ approximant of $T$. For each nonempty subset $\Lambda$ of the complex numbers $\mathbf{C}$, denote by $N(\Lambda)$ the set of all normal operators in $B(H)$ with spectrum included in $\Lambda$. Such sets are studied by P. R. Halmos in [2], where it is shown that if $\Lambda$ is closed, then $N(\Lambda)$ is norm closed and, moreover, each normal operator in $B(H)$ has an $N(\Lambda)$-approximant. If $H$ is finite dimensional, then an easy compactness argument shows that for any closed set $\Lambda$ the set $N(\Lambda)$ is proximinal in $B(H)$. If $H$ is infinite dimensional, then for $\Lambda=[0, \infty)$ or $\Lambda=$ all real numbers, the set $N(\Lambda)$ is proximinal in $B(H)$ (see [1]), and for $\Lambda=$ the unit circle, the set $N(\Lambda)$ of unitary operators fails to be proximinal in $B(H)$ [5].

The purpose of this note is to show that if $H$ is infinite dimensional, then neither the set of normal operators $(\Lambda=\mathbf{C})$ nor the set of orthogonal projections $(\Lambda=\{0,1\})$ is a proximinal subset of $B(H)$; this latter result answers a question raised in [2].

1. Normal operators. We shall use the following definition: a nonzero vector $f$ in $H$ is called a maximal vector for an operator $X$ if $\|X f\|=\|X\| \cdot\|f\|$. It is not difficult to see that $f$ is a maximal vector for $X$ if and only if $X^{*} X f$ $=\left\|X^{*} X\right\| \cdot f$.

1.1 Lemma. If $f$ is a maximal vector for $X$ and if $g$ is any vector such that $(f, g)=0$, then $(X f, X g)=0$.

Proof. $(X f, X g)=\left(X^{*} X f, g\right)=\left\|X^{*} X\right\| \cdot(f, g)$.

For $T$ in $B(H)$ write $d(T)=\inf \{\|T-N\|: N$ normal $\}$.

Received by the editors January 26, 1976 and, in revised form, March 9, 1976.

AMS (MOS) subject classifications (1970). Primary 47B15; Secondary 41A65.

Key words and phrases. Normal operator, hyponormal operator, projection, proximinal set.

1 This paper is part of the author's Ph. D. thesis written at Indiana University under the direction of Professor P. R. Halmos. 
1.2 THEOREM. If $T$ is an operator with a dense range such that $d(T) \leqslant\|T\| / 2$ and such that the kernel of $T$ contains a maximal vector for the adjoint of $T$, then $T$ fails to have a normal approximant.

Proof. Assume, without loss of generality, that $\|T\|=1$, and let $f$ be a unit vector such that $T f=0$ and $\left\|T^{*} f\right\|=1$. It follows, as in [3] and [4], that these properties of $f$ imply $d(T) \geqslant \frac{1}{2}$; hence $d(T)=\frac{1}{2}$ by the hypothesis on $d(T)$. We now show that not only is there no normal operator at this distance from $T$, but also there is no hyponormal operator $N$ such that $\|N-T\| \leqslant \frac{1}{2}$ (an operator $N$ is hyponormal if $N^{*} N \geqslant N N^{*}$ ).

Suppose there exists a hyponormal operator $N$ such that $\|N-T\| \leqslant \frac{1}{2}$. Then $N^{*} f=T f^{*} / 2$ because $\left\|N^{*} f\right\| \leqslant\|N f\|=\|N f-T f\| \leqslant \frac{1}{2}$ and also $\left\|N^{*} f-T^{*} f\right\| \leqslant \frac{1}{2}$; the only vector in $H$ satisfying both these inequalities is $T^{*} f / 2$. Hence $\left\|N^{*} f\right\|=\|N f\|=\frac{1}{2}$.

We shall now obtain a contradiction by proving $N f=0$. Observe that $N^{*} N f=N N^{*} f$ since $\|N f\|=\left\|N^{*} f\right\|$ and $N$ is hyponormal. Assertion: if $g$ is any vector such that $(f, g)=0$, then $(N f, T g)=0$. Proof:

$$
\begin{aligned}
(N f, T g) & =(N f, N g)-(N f,(N-T) g) \\
& =(N f, N g)-((N-T) f,(N-T) g) \text { because } T f=0 \\
& =(N f, N g) \text { because } f \text { is maximal for } N-T \text { and Lemma } 1.1 \text { applies } \\
& =\left(N^{*} f, N^{*} g\right) \text { since } N^{*} N f=N^{*} f \\
& =\left(T^{*} f / 2, N^{*} g\right) \text { since } N^{*} f=T^{*} f / 2 \\
& =\left(T^{*} f / 2, N^{*} g\right)-\left(T^{*} f / 2, T^{*} g\right) \text { because } f \text { is maximal for } T^{*} \\
& =\left(T^{*} f / 2,\left(N^{*}-T^{*}\right) g\right) \\
& =-\left(\left(N^{*}-T^{*}\right) f,\left(N^{*}-T^{*}\right) g\right) \text { because } T^{*} f / 2=-\left(N^{*}-T^{*}\right) f \\
& =0 \text { because } f \text { is maximal for } N^{*}-T^{*} .
\end{aligned}
$$

Thus $(N f, T g)=0$ if $(f, g)=0$. Because also $T f=0$, we can conclude that $N f$ is orthogonal to the range of $T$. Because the range of $T$ is dense, this implies $N f=0$. Hence no such hyponormal operator $N$ exists. This proves Theorem 1.2 .

1.3 EXAMPLE. The hypotheses of Theorem 1.2 are satisfied by the operator $T=$ the adjoint of the unilateral weighted shift with weight sequence $(1,1 / 2,1 / 3, \ldots, 1 / n, \ldots)$.

To see this, let $\left\{e_{1}, e_{2}, \ldots\right\}$ be an orthonormal basis of $H$ such that $T^{*} e_{s}=e_{s+1} / s$, for $s=1,2, \ldots$ Clearly the range of $T$ is dense, and $e_{1}$ is a maximal vector for $T^{*}$ that is in the kernel of $T$. To show $d(T) \leqslant \frac{1}{2}$, define the normal operators $N_{k}$ for $k=2,3, \ldots$ by $N_{k} e_{1}=e_{k} / 2, N_{k} e_{s}=e_{s-1} / 2$ for $2 \leqslant s \leqslant k$ and $N_{k} e_{s}=e_{s} / 2$ for $s>k$. Then $N_{k}$ is normal $\left(2 N_{k}\right.$ is a unitary operator) and $\left\|N_{k}-T\right\| \leqslant(1 / 2)+(1 / k)$. Thus $d(T) \leqslant \frac{1}{2}$. This inequality also follows from [4, Theorem 3$]$ since $T$ is compact. 
2. Projections. In this section we show that if $H$ is infinite dimensional, then the set of orthogonal projections is not proximinal in $B(H)$. We prove, in fact, the following more general result.

2.1 THEOREM. If $\Lambda$ is a nonempty compact subset of the real line, then $N(\Lambda)$ is proximinal if and only if $\Lambda$ is an interval.

Proof. If $\Lambda$ is a closed interval, then $N(\Lambda)$ is closed in the weak operator topology and hence is proximinal (see [1, p. 956]).

Conversely, assume $\Lambda$ is not an interval. Notice that if $\Lambda^{\prime}=\{\alpha \lambda+\beta$ : $\lambda$ in $\Lambda\}$ is the image of $\Lambda$ under the affine transformation $\alpha \lambda+\beta$ for some (fixed) numbers $\alpha$ and $\beta$ with $\alpha \neq 0$, then $N\left(\Lambda^{\prime}\right)$ is proximinal if and only if $N(\Lambda)$ is proximinal. Hence we assume that -1 and +1 are both in $\Lambda$ and $|\lambda| \geqslant 1$ for all $\lambda$ in $\Lambda$. Write $a=\min \Lambda$ and $b=\max \Lambda$, so that $\Lambda \subseteq[a,-1]$ $\cup[1, b]$. Choose $c>0$ and let $\left\{e_{1}, e_{2}, e_{3}, \ldots\right\}$ be an orthonormal basis for $H$. With respect to the decomposition $\operatorname{span}\left\{e_{1}, e_{2}, e_{3}\right\} \oplus \operatorname{span}\left\{e_{4}, e_{5}, e_{6}, \ldots\right\}$, define $A$ in $B(H)$ by

$$
A=\left(\begin{array}{ccc}
a & i c & 0 \\
i c & 0 & i c \\
0 & i c & b
\end{array}\right) \oplus \operatorname{diag}(1,1 / 2,1 / 3, \ldots, 1 / n, \ldots)
$$

Assertion: The operator $A$ fails to have an $N(\Lambda)$-approximant. Proof: Notice first that dist $(A, N(\Lambda))=\left(1+2 c^{2}\right)^{\frac{1}{2}}$. To see this, let $N$ be in $N(\Lambda)$. Then

$$
\begin{aligned}
\|N-A\|^{2}+\left\|N-A^{*}\right\|^{2} & \geqslant\left\|(N-A) e_{2}\right\|^{2}+\left\|\left(N-A^{*}\right) e_{2}\right\|^{2} \\
& =\left\|(N-A) e_{2}\right\|^{2}+\left\|(N+A) e_{2}\right\|^{2} \\
& =2\left(\left\|N e_{2}\right\|^{2}+\left\|A e_{2}\right\|^{2}\right) \geqslant 2\left(1+2 c^{2}\right) .
\end{aligned}
$$

To prove the reverse inequality, define $N_{k}$ in $N(\Lambda)$ for $k=4,5, \ldots$ by $N_{k} e_{1}=a e_{1}, N_{k} e_{2}=e_{k}, N_{k} e_{3}=b e_{3}, N_{k} e_{k}=e_{2}$ and $N e_{j}=e_{j} \quad$ otherwise; then $\left\|N_{k}-A\right\| \leqslant\left(1+2 c^{2}\right)^{\frac{1}{2}}+(1 /(k-3))$. Thus

$$
\operatorname{dist}(A, N(\Lambda))=\left(1+2 c^{2}\right)^{\frac{1}{2}} .
$$

Now suppose there exists an $N$ in $N(\Lambda)$ such that $\|N-A\|=\left(1+2 c^{2}\right)^{\frac{1}{2}}$. The arguments above show that in this case $e_{2}$ is a maximal vector for both $N-A$ and $N-A^{*}$ and $\left\|N e_{2}\right\|=1$. We now obtain a contradiction by proving $\mathrm{Ne}_{2}=0$.

We show first that $N e_{2}$ is orthogonal to $\left\{e_{1}, e_{2}, e_{3}\right\}$. Let $N$ have the matrix representation $\left(x_{s t}\right)$ with respect to the orthonormal basis $\left\{e_{1}, e_{2}, \ldots\right\}(s, t$ $\left.=1,2, \ldots ; x_{s t}=\bar{x}_{t s}\right)$. Lemma 1.1 implies

$$
\left((N-A) e_{1},(N-A) e_{2}\right)=0=\left(\left(N-A^{*}\right) e_{1},\left(N-A^{*}\right) e_{2}\right) .
$$

Hence 


$$
\left(x_{11}-a\right)\left(x_{21}+i c\right)+\left(x_{21}-i c\right) x_{22}+x_{31}\left(\bar{x}_{32}+i c\right)+\sum_{s=4}^{\infty} x_{s 1} \bar{x}_{s 2}=0
$$

and

$$
\left(x_{11}-a\right)\left(x_{21}-i c\right)+\left(x_{21}+i c\right) x_{22}+x_{31}\left(\bar{x}_{32}-i c\right)+\sum_{s=4}^{\infty} x_{s 1} \bar{x}_{s 2}=0 .
$$

Subtracting these two equations and dividing by $2 i c$ yields $\left(x_{11}-a\right)-x_{22}$ $+x_{31}=0$.

We can also conclude from Lemma 1.1 that

$$
\left((N-A) e_{2},(N-A) e_{3}\right)=0=\left(\left(N-A^{*}\right) e_{2},\left(N-A^{*}\right) e_{3}\right) .
$$

Hence

$$
\left(\bar{x}_{21}-i c\right) x_{31}+x_{22}\left(x_{32}+i c\right)+\left(x_{32}-i c\right)\left(x_{33}-b\right)+\sum_{s=4}^{\infty} x_{s 2} \bar{x}_{s 3}=0
$$

and

$$
\left(\bar{x}_{21}+i c\right) x_{31}+x_{22}\left(x_{32}-i c\right)+\left(x_{32}+i c\right)\left(x_{33}-b\right)+\sum_{s=4}^{\infty} x_{s 2} \bar{x}_{s 3}=0 .
$$

Subtracting these two equations and dividing by $-2 i c$ yields $\left(x_{33}-b\right)-x_{22}$ $+x_{31}=0$.

From these observations we can conclude that $x_{11}-a=x_{22}-x_{31}=x_{33}$ $-b$; thus $x_{33}-x_{11}=b-a$. Hence $x_{11}$ and $x_{33}$ are two points in the numerical range of $N$ that are as far apart as any two points in the numerical range can be (since the spectrum of $N$ is included in $[a, b]$ ); this implies that $x_{11}=a$ and $x_{33}=b$ and consequently $x_{22}=x_{31}$. Since extreme points in the numerical range of $N$ must be eigenvalues, we conclude also that $N e_{1}=a e_{1}$ and $\mathrm{Ne}_{3}=\mathrm{be}_{3}$.

Since $N e_{1}=a e_{1}$, we have $x_{12}=\bar{x}_{21}=0$ and $x_{22}=x_{31}=0$; since $N e_{3}$ $=b e_{3}$, we have $x_{32}=\bar{x}_{23}=0$. Thus $N e_{2}$ is orthogonal to $\left\{e_{1}, e_{2}, e_{3}\right\}$.

It now remains to show $x_{t 2}=0$ for $t=4,5, \ldots$. Since $\left\|N e_{2}\right\|=1$ and $N$ is bounded below by 1 , it follows that $N^{2} e_{2}=e_{2}$; thus $\left(N e_{2}, N e_{t}\right)=0$ for $t \neq 2$. Since $x_{12}=x_{22}=x_{32}=0$, it follows that $\left(N e_{2}, N e_{t}\right)=\sum_{s=4}^{\infty} x_{s 2} \bar{x}_{s t}$ $=0$.

Because $e_{2}$ is maximal for $N-A$, it also follows that $\left((N-A) e_{2},(N-A) e_{t}\right)$ $=0$. This implies for $t \geqslant 4$ that

$$
0=\left((N-A) e_{2},(N-A) e_{t}\right)=\left(\sum_{s=4}^{\infty} x_{s 2} \bar{x}_{s t}\right)-\frac{x_{t 2}}{t-3}
$$

because $A e_{t}=e_{t} /(t-3)$ and $x_{1 t}=x_{3 t}=x_{22}=0$. Using the fact that $\sum_{s=4}^{\infty} x_{s 2} \bar{x}_{s t}=0$, we can thus conclude that $x_{t 2}=0$ for $t=4,5, \ldots$ Hence $\mathrm{Ne}_{2}=0$. This proves Theorem 2.1.

The preceding results contribute to a characterization of those sets $\Lambda$ for 
which $N(\Lambda)$ is proximinal in $B(H)$. The only sets $\Lambda$ for which it is known that $N(\Lambda)$ is proximinal are $\Lambda=$ one point, $\Lambda=[0,1], \Lambda=[0, \infty), \Lambda=(-\infty$, $\infty)$, or an affine translation of one of these intervals; these sets $N(\Lambda)$ are all closed in the weak operator topology and are thus proximinal. It would be interesting to determine whether there are any other sets $\Lambda$ for which $N(\Lambda)$ is proximinal.

It is not difficult to see that if $N(\Lambda)$ is proximinal, then $\Lambda$ must have empty interior. Proof: If $\Lambda$ includes an open set, then (by using an affine transformation $\alpha \lambda+\beta$ if necessary) we can assume that $\Lambda$ includes the circle of radius $\frac{1}{2}$ with center at the origin. Hence the operators $N_{k}$ in the example in $\S 1$ are in $N(\Lambda)$, and the weighted shift in that example fails to have an $N(\Lambda)$ approximant.

Thus int $(\Lambda)=\varnothing$ is a necessary condition for $N(\Lambda)$ to be proximinal. This condition is satisfied, of course, by subsets of the real line, but even in this special case the problem is not solved. In particular, for $\Lambda=(-\infty,-1]$ $\cup[1, \infty)$ it is apparently not known whether $N(\Lambda)$ is proximinal.

\section{REFERENCES}

1. P. R. Halmos, Positive approximants of operators, Indiana Univ. Math. J. 21 (1971/72), 951-960. MR 45 \#919.

2. - Spectral approximants of normal operators, Proc. Edinburgh Math. Soc. (2) 19 (1974/75), 51-58. MR 49 \#9674.

3. P. R. Halmos and J. E. McLaughlin, Partial isometries, Pacific J. Math. 13 (1963), 585-596. MR 28 \#477.

4. R. B. Holmes, Best approximation by normal operators, J. Approximation Theory 12 (1974), 412-417. MR 50 \#128.

5. D. D. Rogers, Approximation by unitary and essentially unitary operators, Acta Sci. Math. (Szeged) (to appear).

Department of Mathematics, University of Georgia, Athens, Georgia 30602

Current address: Mathematics Department, U.S. Naval Academy, Annapolis, Maryland 21402 